\title{
Exploring Nociceptive Detection Thresholds Combined with Evoked Potentials in Patients with Diabetes Mellitus
}

\author{
Tom Berfelo, Imre P. Krabbenbos, Boudewijn van den Berg, Silvano R. Gefferie, Jan R. Buitenweg
}

\begin{abstract}
There is a lack of diagnostic tools that can objectively measure small fiber neuropathy (SFN) in patients with diabetes mellitus (DM). Recently, nociceptive nerve function was observed by nociceptive detection thresholds (NDTs) and brain evoked potentials (EPs) during intraepidermal electrical stimulation (IES) targeting A $\delta$-fibers. In this proof of principle, we studied whether it is possible to measure NDTs combined with EPs in DM patients with and without neuropathic pain. Furthermore, we explored the sensitivity of NDTs and EPs for polyneuropathy in these patients. Five DM patients diagnosed with painful neuropathy $(D M p)$, five DM patients without painful neuropathy $(D M)$, and five healthy controls $(H C)$ were analyzed. These preliminary results showed that we can accurately measure NDTs and EPs in patients with diabetes. Strikingly, increased NDTs were found in DM and DMp compared to HC, of which the DMp showed the largest NDTs. This suggests that NDTs during IES could be a powerful biomarker for monitoring peripheral dysfunctions. Current EEG data of patients did not show any significant differences. The population needs to be enlarged before we can investigate the sensitivity of these NDTs and EPs to diabetic polyneuropathy and associated changes in nociceptive processing in more detail.
\end{abstract}

Clinical Relevance - This proof of principle in a small group of patients with diabetes mellitus potentially treats the observation of the loss of nociceptive function occurring with small fiber neuropathy. That helps the development of a diagnostic measure to monitor future (early-stage) nociceptive dysfunctions in a clinical environment.

\section{INTRODUCTION}

Peripheral polyneuropathy is a common condition in patients with diabetes mellitus (DM), often manifested symmetrically in the hands and feet, characterized by a loss of sensory function. The irreversible damage of peripheral nerve fibers is caused by enhanced blood glucose concentration [1]. After a sustained period of nerve damage, approximately 20 to $30 \%$ of the patients with diabetic polyneuropathy (DPN) develops chronic painful peripheral polyneuropathy [2]. There is increasing evidence that the central nervous system is involved in the development of central sensitization in patients with diabetes as a result of the changed nociceptive input due to damaged small nerve fibers (i.e., $\mathrm{A} \delta$ and c-fibers) $[3,4]$. Unfortunately, no effective treatment is available for patients with diabetes and neuropathic pain [5]. Objective assessment of small fiber neuropathy (SFN) is still challenging in clinical

Research supported by the Netherlands Organization for Scientific Research (NWO) and the Anesthesiology R\&D department of the St. Antonius Hospital Nieuwegein.

T. Berfelo, B. van den Berg, and J.R. Buitenweg are with the department of Biomedical Signals and Systems, Technical Medical Centre, University of Twente, Enschede, the Netherlands (e-mail: t.berfelo@utwente.nl). practice. Identifying early-stage small fiber dysfunctions in patients with diabetes might contribute to early glycemic control, which avoids the later stage complications.

Recently, we have developed the NDT-EP method for observing psychophysical and neurophysiological effects in response to intra-epidermal electrical stimulation (IES) [6]. This method measures nociceptive detection thresholds (NDTs) and brain evoked potentials (EPs) by activating nociceptive $A \delta$-nerve fibers in the skin using intra-epidermal electrical stimuli around the detection threshold. A mix of stimuli with different properties can be applied to the subject. Subsequently, the effect of stimulus properties on the detection probability and the EP can be quantified using a generalized linear (mixed) model [7]. Outcome parameters, such as the NDT and stimulus-related EP can be used for a mechanismbased approach of peripheral and central nerve function.

Using the NDT-EP method, we have shown that several nociceptive processing steps, including peripheral nerve fiber recruitment, central synaptic summation, and habituation to repetitive administered trials, can be quantified in healthy subjects [8]. Also, we have demonstrated that these results can be replicated in a clinical environment and that it is sensitive to observe phenomena of altered central nociceptive processing in chronic pain patients, such as failed back surgery syndrome [9]. The next step is to investigate whether the NDTEP method has the potential to detect (early-stage) peripheral nociceptive dysfunctions in patients with SFN.

In this proof of principle, we firstly explore the feasibility of the NDT-EP method in patients with diabetes. Peripheral dysfunctions are hypothesized to be observed and possible central nociceptive alterations might be monitored using EEG. The patients with diabetes will be subdivided into two groups: (1) DM patients diagnosed with painful neuropathy and (2) DM patients without painful neuropathy. Psychophysical measures (i.e., nociceptive detection rate, thresholds, and slopes of the psychophysical curve) and EEG activity (i.e., grand average EP, signal-to-noise ratio, and P2 amplitudes) will be explored, and subsequently, be compared with results from healthy controls.

\section{METHODS}

The study was approved by the Medical Research Ethics Committees United (MEC-U, file-number: NL66136.100.18) and subjects signed an informed consent.

T. Berfelo, I.P. Krabbenbos, and S.R. Gefferie are with the Department of Anesthesiology, Intensive Care and Pain Medicine, St. Antonius Hospital, Nieuwegein, the Netherlands. 


\section{A. Subjects}

Three groups were enrolled in this explorative study, which consisted of (1) five DM patients diagnosed with painful neuropathy $(D M p),(2)$ five $\mathrm{DM}$ patients without painful neuropathy $(D M)$, and (3) five healthy controls $(H C)$. The DM and $\mathrm{HC}$ were included if they had an NRS-score of 0.0 and did not have a diagnosed DPN by a neurologist, or history of chronic pain. In contrast, the DMp was diagnosed with DPN and suffered at least for 3 months from neuropathic pain. All subject's ages ranged from 18 to 75 years. Subjects were excluded when they suffered from any other neurological disorder.

All subjects underwent one measurement session at the St. Antonius Hospital Nieuwegein, which comprises a questionnaire, neurological examination (only for patients with diabetes), and two measurements using the NDT-EP method (i.e., stimulating the dominant and non-dominant hand).

\section{B. Procedure}

During the NDT-EP method, the subjects sat in a comfortable chair holding an AmbuStim 1-channel (NociTRACK B.V.) in their hand, which was connected to two stimulation electrodes on the dorsum of the other hand. A cathodic 5-needle electrode (with $0.5 \mathrm{~mm}$ needles) was attached distally from an anodic 9x5 cm TENS electrode [10]. A mix of intra-epidermal electrical stimuli was applied around the detection threshold to preferentially activate nociceptive A $\delta$-fibers using the 5neelde electrode. Three types of $210 \mu$ s square wave stimuli were selected: (1) single-pulse (SP), (2) double-pulse stimuli with $10 \mathrm{~ms}$ inter-pulse interval (DP10), and (3) double-pulse stimuli with $40 \mathrm{~ms}$ inter-pulse interval (DP40). The subjects were instructed to press-and-hold the button of the stimulator, and to release it when they detected a sensation which they ascribed to the stimulator. Before the subjects started the NDTEP method, they were familiarized with the stimuli using a normal staircase procedure with a step size of $0.05 \mathrm{~mA}$ to determine an initial detection threshold for each stimulus type. Subsequently, an advanced stimulus selection procedure was performed to randomly administer the three stimulus types around the detection threshold [11]. For each stimulus, a vector of 5 amplitudes was initialized around the initial detection threshold with a step size of $0.025 \mathrm{~mA}$. One value was randomly selected from the 5 amplitudes and used for stimulation. When a stimulus was not detected, the vector of amplitudes was increased with a fixed step of $0.025 \mathrm{~mA}$, but when a stimulus was detected, the vector of amplitudes was decreased by $0.025 \mathrm{~mA}$. This procedure was continued for a total amount of 450 stimuli (i.e., 150 per stimulus type) during each measurement (Figure 1).

Simultaneously, stimulus-related brain EPs were recorded with a sampling rate of $1000 \mathrm{~Hz}$. This was performed using an ANT Neuro Waveguard EEG cap (10-20 system) with 64 $\mathrm{Ag} / \mathrm{Cl}$ electrodes. The cap was connected to a TMSi 72channel Refa EEG amplifier. Electrode impedances were kept below $5 \mathrm{kOhm}$.

\section{Nociceptive Detection Rate, Thresholds, and Slopes}

Individual average detection rate $\left(R_{d e t}\right)$, nociceptive detection thresholds $\left(T_{S P}, T_{D P 10}, T_{D P 40}\right)$, and slopes $\left(S_{S P}, S_{D P 10}, S_{D P 40}\right)$ were calculated from the 450 collected stimulus-response pairs

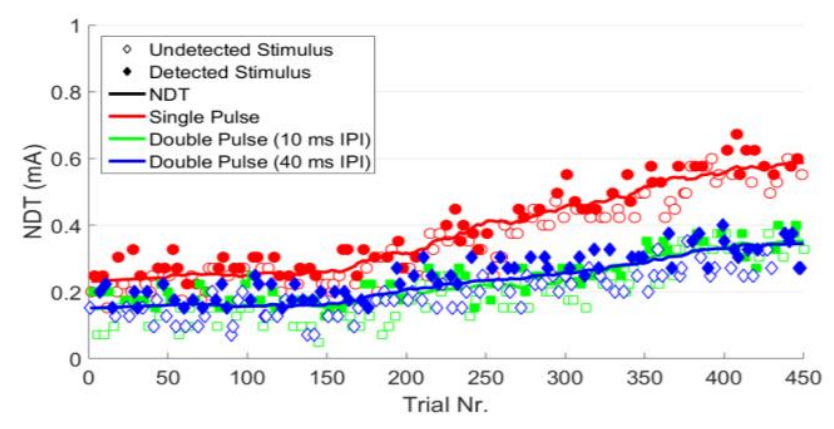

Figure 1. An example of psychophysical detection thresholds extracted from one single measurement of a healthy subject. Detection thresholds of three different stimulus types (i.e., single pulse, double-pulse with 10 interpulse interval, and double-pulse with $40 \mathrm{~ms}$ inter-pulse interval) were tracked using an adaptive multiple threshold tracking paradigm. In this way, altered nociceptive processing can be quantified in patients with diabetes, in response to multiple stimulus properties. Note that psychophysical change in sensitivity with respect to the trial number $(T R L)$ is included as a fixed effect in the regression models in equation (1) and (2).

(i.e., detected and non-detected) per measurement. A psychophysical curve was determined, in which the detection probability $(P)$ was described as a function of the model intercept, pulse amplitudes $\left(P U 1, P U 2_{10}, P U 2_{40}\right)$ and trial number (TRL) using logistic regression in equation (1). The NDT was defined as the stimulus amplitude at which the detection probability is 0.5 . Individual average NDTs and slopes were computed based on the logistic regression coefficients. The effect of both diagnoses (DIAG) (i.e., DM and DMp) on NDTs and slopes was computed with a linear model using log-transformed variables and assessed for significance using a t-test.

$\ln \left(\frac{P}{1-P}\right) \sim 1+P U 1 * D I A G+P U 2_{10} * D I A G+P U 2_{40} * D I A G+$ $T R L * D I A G$

\section{Evoked Potentials}

The EEG signals were preprocessed using FieldTrip [12], a toolbox in MATLAB (version 2015b; The MathWorks Inc, Natick, Massachusetts, US). A band-pass filter was applied from 0.1 to $40 \mathrm{~Hz}$. A $1.5 \mathrm{~s}$ time window around the administered stimuli (i.e., $0.5 \mathrm{~s}$ pre-stimulus to $1.0 \mathrm{~s}$ poststimulus) was extracted from the EEG signal. Eye blinks and muscular activity were removed from the EEG by independent component analysis. EP signals were derived from the $\mathrm{CPz}$ A1A2 derivation, which was in line with the previous studies $[8,9]$. From this derivation, a grand average EP and the P2 latency were computed. The P2 was defined as the most positive peak between 300 and $500 \mathrm{~ms}$ post-stimulus. At this point, the individual mean P2 amplitude $(\mu(P 2))$ and the individual standard deviation of the $\mathrm{P} 2$ amplitude $(\sigma(P 2))$ were calculated per group using linear regression, as shown in equation (2). The P2 was calculated as a function of the intercept, stimulus amplitudes $\left(P U 1, P U 2_{10}, P U 2_{40}\right)$, trial number $(T R L)$, and stimulus detection $(D)$ (i.e., detected and non-detected stimuli). The signal-to-noise ratio $(S N R)$ was computed based on the mean EEG activity over all trials per measurement, with the EEG value at the P2 latency divided by the standard deviation of the mean EEG baseline ( -0.5 to $0 \mathrm{~s})$.

$P 2_{E E G} \sim 1+P U 1 * D I A G+P U 2_{10} * D I A G+P U 2_{40} * D I A G+$

$T R L * D * D I A G$ 


\section{RESULTS}

Five DM patients diagnosed with painful neuropathy (4 males; age: $66.0 \pm 5.2$ ), five DM patients without painful neuropathy (4 males; age: $59.2 \pm 14.8$ ), and five healthy controls (2 males; age: $43.0 \pm 11.6)$ were included for data analysis. The average DMp patient (CSI-score: $43.6 \pm 14.3$ ) suffered from a central sensitization syndrome, while it did not occur in the DM group (CSI-score: $16.8 \pm 7.5$ ) and HC (CSI-score: $12.0 \pm 8.1$ ). The average duration of DPN in DMp was $7.0 \pm 2.7$ years, with pain duration of $6.8 \pm 2.0$ years, and an NRS-score of $4.2 \pm 1.6$ during the measurements. The duration of diabetes was $14.0 \pm$ 7.1 years in DM and $11.0 \pm 4.8$ years in DMp.

\section{A. Nociceptive Detection Rate, Thresholds, and Slopes}

Individual detection rate $\left(R_{\text {det }}\right)$, nociceptive detection thresholds $\left(T_{S P}, T_{D P 10}, T_{D P 40}\right)$, and slopes $\left(S_{S P}, S_{D P 10}, S_{D P 40}\right)$ of the psychophysical curve are depicted in Figure 2. Individual values differed significantly $(p<0.001)$ between groups. The detection rate seemed to be lower in DM than in $\mathrm{HC}$ and was even significantly $(p<0.001)$ decreased in DMp. Significant larger NDTs were found in DM compared to HC, with the largest NDT values in DMp. Also, the steepness of the slope was significantly decreased over the groups.
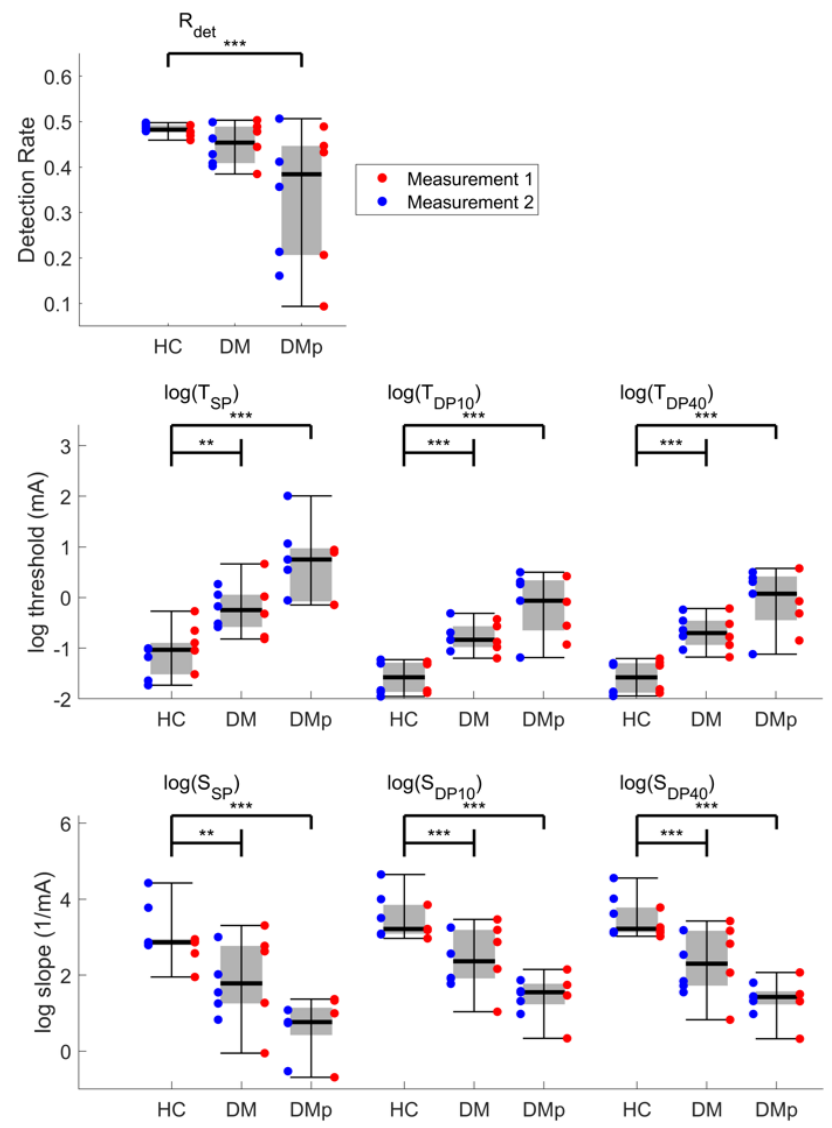

Figure 2. Individual psychophysical detection rate $\left(R_{\text {det }}\right)$, and derived log-transformed detection thresholds $\left(T_{S P}, T_{D P 10}, T_{D P 40}\right)$ and slopes $\left(S_{S P}, S_{D P 10}, S_{D P 40}\right)$ of the psychophysical curve from five subjects of each group (i.e., $\mathrm{HC}$, DM, and $\mathrm{DMp}$ ). The detection rate, threshold, and slopes were averaged over the entire measurement. These values were shown for measurements 1 and 2. Significant altered psychophysical values were seen in patients with diabetes compared to healthy controls $\left({ }^{* *} p<.01\right.$, ${ }^{* * *} p<$ .001). This was found for all stimulus types.

\section{B. Brain Evoked Potentials}

The P2 peak was found at $450 \mathrm{~ms}$ post-stimulus (Figure 3). SNR of the P2 seems to be decreased in DM and DMp but was still good for EEG analysis. Although the variation of the EP decreased for DM with a $p$-value below 0.05 , the mean P2 was not significantly different between groups.
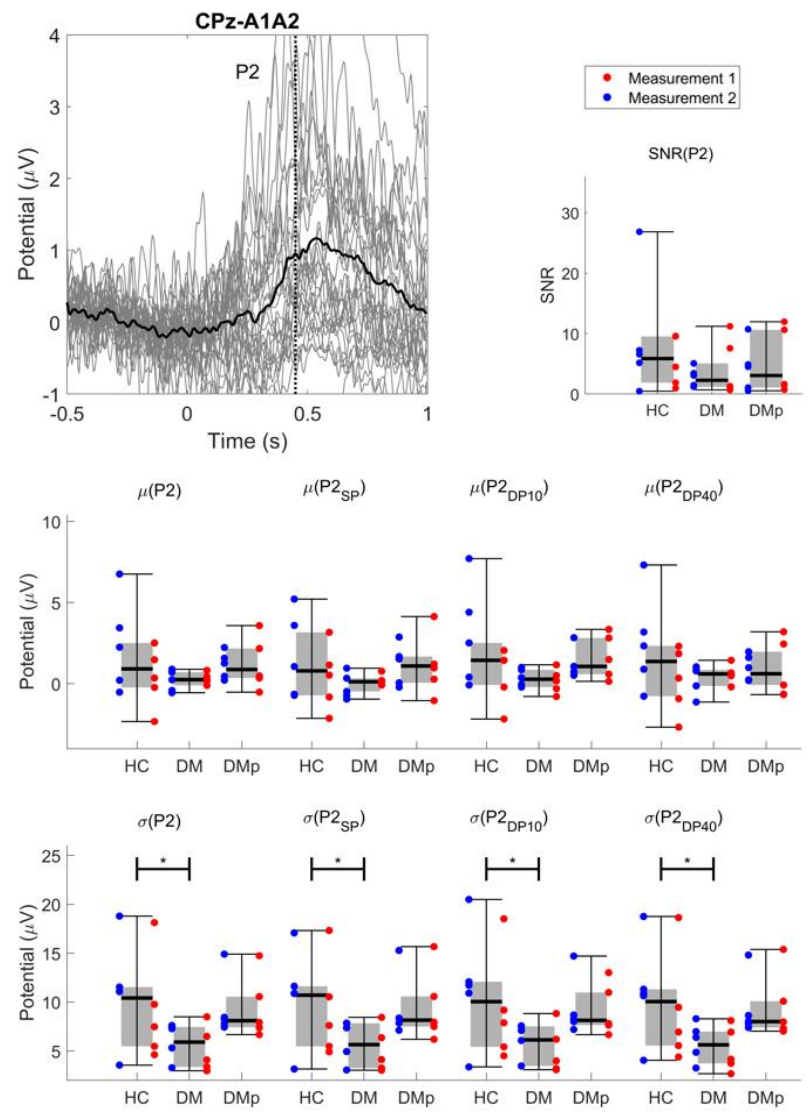

Figure 3. Individual EPs and the grand average EP (bold) from 30 measurements (i.e., two measurements per subject) with a P2 latency determined at $450 \mathrm{~ms}$ post-stimulus. The mean $(\mu)$ of the P2 amplitude did not differ significantly between groups. The average standard deviation $(\sigma)$ of the P2 amplitude was different between DM and $\mathrm{HC}(* p<.05)$.

\section{DISCUSSION}

This proof of principle utilized the NDT-EP method in a small sample of patients with diabetes as a technical pilot. We aimed to explore the feasibility of using the NDT-EP method in five DM patients diagnosed with painful neuropathy and five DM patients without painful neuropathy. Psychophysical outcomes and stimulus-related EPs were compared with results from healthy controls.

Here, we found that the NDT-EP method can be applied to patients with diabetes. We observed lower nociceptive detection rates, higher NDTs, and lower psychophysical slopes in DM compared to HC (Figure 2). Strikingly, these patients were not diagnosed with diabetic polyneuropathy. This implies that an early-stage small fiber dysfunction might be observed by the NDT-EP method. Another research group confirmed that IES of small nerve fibers can be a useful technique for the early detection of diabetic polyneuropathy [13]. 
Moreover, we found that the psychophysical values were more altered in DMp (Figure 2). Note that these patients were exposed to peripheral nerve damage for a similar period as the DM patients (without polyneuropathy). Obviously, the DMp patients showed higher ages than the DM patients. Reduction of nerve fiber density due to age cannot be ruled out.

The EEG activity of patients with diabetes was not significantly different from $\mathrm{HC}$ but the $\mathrm{P} 2$ amplitude seems to be decreased in both DM and DMp (Figure 3). Unfortunately, we did not obtain many detected stimulus-related EPs. This occurred due to the decreased nociceptive detection rate and psychophysical slope among patients. Presumably, the EP will be more accurately computed with an enlarged data set. Then, relationships between stimulus-properties and the EP can be explored using linear mixed regression. Since the increased CSI-score reflected central sensitization syndrome in DMp on a group-level, the correlation between clinical features (e.g., CSI-score) and EP components should be investigated.

Current groups were heterogeneously distributed based on subject characteristics (e.g., age and sex) and clinical features (e.g., duration of disease). The participants should be matched on subject characteristics and enlarged to twenty subjects each before our results can be interpreted in detail.

\section{CONCLUSION}

To conclude, it was possible to measure nociceptive detection thresholds, slope, and evoked potentials using the NDT-EP method in patients with diabetes. The changed nociceptive detection rate, thresholds, and psychophysical slopes in DM and DMp patients implicate that the described method of testing can be promising to observe altered nociceptive processing in patients with diabetes. The added value of EEG cannot be determined yet due to the small data set. Therefore, it is recommended to enlarge the group and investigate the sensitivity of the NDT-EP method to observe altered nociceptive processing in patients with (early-stage) peripheral dysfunctions.

\section{REFERENCES}

[1] Callaghan BC, Little AA, Feldman EL, Hughes RAC. (2012). Enhanced glucose control for preventing and treating diabetic neuropathy. Cochrane Database of Systematic Reviews 2012, Issue 6. Art. No.: CD007543. DOI: 10.1002/14651858.CD007543.pub2.

[2] Juster-Switlyk, K., \& Smith, A. G. (2016). Updates in diabetic peripheral neuropathy. F1000Research, 5, F1000 Faculty Rev-738. https://doi.org/10.12688/f1000research.7898.1

[3] Aslam, Amir \& Singh, Jaipaul \& Rajbhandari, Satyan. (2014). Pathogenesis of Painful Diabetic Neuropathy. Pain research and treatment. 2014. 412041. 10.1155/2014/412041.

[4] Nedergaard RB, Nissen TD, Mørch CD, Meldgaard T, Juhl AH, Jakobsen PE, Karmisholt J, Brock B, Drewes AM, Brock C. (2020). Diabetic Neuropathy Influences Control of Spinal Mechanisms. J Clin Neurophysiol. doi: 10.1097/WNP.0000000000000691. Epub ahead of print. PMID: 32501945.

[5] Javed, S., Petropoulos, I. N., Alam, U., \& Malik, R. A. (2015). Treatment of painful diabetic neuropathy. Therapeutic Advances in Chronic Disease, 15-28. https://doi.org/10.1177/2040622314552071

[6] Van den Berg, B., Doll, R. J., Mentink, A. L. H., Siebenga, P. S., Groeneveld, G. J., \& Buitenweg, J. R. "Simultaneous tracking of psychophysical detection thresholds and evoked potentials to study nociceptive processing". Behavior Research Methods, vol. 52, pp.
1617-1628, 2020. doi: https://www.doi.org/10.3758/s13428-01901338-7

[7] B. van den Berg and J. R. Buitenweg, "Analysis Of Nociceptive Evoked Potentials During Multi-Stimulus Experiments Using Linear Mixed Models," 2018 40th Annual International Conference of the IEEE Engineering in Medicine and Biology Society (EMBC), Honolulu, HI, USA, 2018, pp. 3048-3051, doi: 10.1109/EMBC.2018.8513032

[8] Van den Berg, B., \& Buitenweg, J. R. (2021). Observation of Nociceptive Processing: Effect of Intra-Epidermal Electric Stimulus Properties on Detection Probability and Evoked Potentials. Brain Topography. doi: 10.1007/s10548-020-00816-y

[9] Tom Berfelo, Boudewijn van den Berg, Imre P. Krabbenbos, Jan R. Buitenweg (2019). Observing Electrical Brain Responses around the Nociceptive Detection Threshold. 11th Congress of the European Pain Federation EFIC, Valencia, Spain.

[10] Steenbergen P, Buitenweg JR, Trojan J, van der Heide EM, van den Heuvel T, Flor H, Veltink PH. A system for inducing concurrent tactile and nociceptive sensations at the same site using electrocutaneous stimulation. Behav Res Methods. 2012 Dec;44(4):924-33. doi: 10.3758/s13428-012-0216-y. PMID: 22806702; PMCID: PMC3509324.

[11] Doll RJ, Buitenweg JR, Meijer HG, Veltink PH. (2014) Tracking of nociceptive thresholds using adaptive psychophysical methods. Behavior Research Methods, 46(1):55-66. DOI: 10.3758/s13428-0130368-4.

[12] Oostenveld, R., Fries, P., Maris, E., \& Schoffelen, J. M. "FieldTrip: Open source software for advanced analysis of MEG, EEG, and invasive electrophysiological data". Computational Intelligence and Neuroscience, 2011. doi: 10.1155/2011/156869

[13] Kukidome, D., Nishikawa, T., Sato, M., Igata, M., Kawashima, J., Shimoda, S., Matsui, K., Obayashi, K., Ando, Y., \& Araki, E. (2016). Measurement of small fibre pain threshold values for the early detection of diabetic polyneuropathy. Diabetic medicine: a journal of the British Diabetic Association, 33(1), 62-69. https://doi.org/10.1111/dme.12797 